\title{
GRAVIDEZ NA ADOLESCÊNCIA: ESTUDO COMPARATIVO
}

\author{
Fanny Viviana Lopez Alegria* \\ Néia Schor \\ Arnaldo Augusto F. de Siqueira*
}

LOPEZ A., F.V. et al. Gravidez na adolescencia: estudo comparativo. Rev. Saúde públ., S.Paulo, 23:473-7, 1989.

\begin{abstract}
RESUMO: Estudou-se uma populaçăo de 349 mulheres que deram a luz no periodo de 01/05/86 a 31/07/86, num Serviço de Obstetrícia da Grande São Paulo. Os resultados revelam que o grupo de gestantes adolescentes $(22,2 \%)$ teve uma assistência pré-natal deficiente, já que a grande maioria $(\mathbf{7 0 , 6 \% )}$ teve a primeira consulta no segundo trimestre, tendo em média duas consultas. Durante a gestaçăo, este grupo apresentou menor incidencia de patologias com tratamento ambulatorial $(39,3 \%)$ e hospitalar $(7,9 \%)$, em comparaçảo com as gestantes adultas $(44,4 \%$ e $14,7 \%$, respectivamente). Em relação ao tipo de parto, as adolescentes apresentaram maior proporçāo de partos operatórios, sendo $25,7 \%$ por fórceps e $22,9 \%$ cesárea, contra 14,7\% e 19,7\% nas gestantes adultas, respectivamente. Verificou-se nas gestantes adolescentes maior proporção de intercorrências intraparto $(12,9 \%$ contra $8,2 \%$ nas gestantes adultas), predominando neste periodo a hemorragia e a toxemia. Quanto as intercortencias no puerpério, estas se verificaram em $15,7 \%$ das gestantes adolescentes e em $11,8 \%$ das adultas, sendo a anemia pós-parto, a toxemia e a infecção puerperal as patologias mais comuns.
\end{abstract}

DESCRITORES: Gravidez na adolescência. Cuidado pré-natal. Complicaçōes na gravidez.

\section{INTRODUÇĀO}

As mudanças nos padrões de comportamento experimentadas pelos adolescentes nas últimas décadas revelam problemas que repercutem nos aspectos biopsicossociaiis deste grupo, sendo, sem dúvida, a de maior repercussão aquela relacionada aos padrões que envolvem a atividade sexual.

Como conseqüência das mudanças relacionadas ao exercício da sexualidade, pode-se regis-. trar o alarmante aumento no índice de gravidez entre adolescentes. Diversos fatores podem ter contribuído para tanto, entre eles: menarca precoce; casamento tardio; deficiência na educaçăo sexual; estilo de vida urbana e quase total ausência de serviços de saúde especializados em adolescentes $^{2,3,12}$.

Considerada de alto risco ${ }^{3}$, a gravidez na adolescência chamou a atenção de muitos pesquisadores pela maior incidência de intercorrências obstétricas apresentadas nesta faixa etária, quan. do comparadas a outras idades, tais como: toxe- mia, infecção urinária e anemia, que influem diretamente nas taxas de morbimortalidade materna e perinatal ${ }^{1,2,6}$.. Esses estudos revelam que essas mudanças de comportamento não vêm señdo acompanhadas através do tempo pela total satisfação dessas necessidades em nível de serviços de saúde. De acordo com os levantamentos de Friedman e Edströ ${ }^{5}$, bem como o do Ministério da Saúde no Brasi ${ }^{7}$, só ultimamente se tomou consciência dessa problemática em nível de saúde pública, tendo-se desenvolvido as primeiras estratégias de planejamento de serviços de saúde dirigidas aos adolescentes.

Portanto, para atender adequadamente às necessidades de saúde desta população são necessários a identificação e o conhecimento precisos da magnitude dos problemas, para se estabelecer prioridades e traçar projetos adequados e viáveis a nível de assistência à saúde.

O objetivo do presente trabalho é verificar as intercorrências e o tipo de assistência ofereci-

Departamento de Saúde Materno-Infantil da Faculdade de Saúde Pública da Universidade de São Paulo - Av. Dr. Arnaldo, 715 - 01255 - Sảo Paulo, SP - Brasil. 
da, durante o ciclo gravidico-puerperal, às gestantes adolescentes e adultas que recorreram a um serviço de obstetrícia da Grande São Paulo.

\section{POPULAÇĀO E MÉTODOS}

Foi estudado o universo das gestantes que ingressaram num serviço de obstetrícia, localizada em um município da Grande São Paulo, no período de 01/05/86 a 31/07/86, e que tiveram, como resultado da gestação, o parto. A população de estudo foi de 349 gestantes: 77 adolescentes e 272 adultos.

O material utilizado para o estudo compreendeu: prontuário clínico; cartão da gestante; e entrevista feita com a gestante e/ou equipe de saúde, no puerpério imediato.

Dos prontuários clínicos, foram levantadas as seguintes variáveis: idade (em anos completos), patologia(s) apresentada(s) no pré-natal com tratamento ambulatorial, patologia(s) apresentada(s) no pré-natal com hospitalização, tipo de parto, intercorrências no parto e no puerpério.

Do cartão da gestante foram levantadas as vàriáveis: início da assistência pré-natal e número de consultas no pré-natal.

Quando não havia registro de dados no prontuário clínico ou no cartão da gestante, estes foram levantados através de entrevista com a paciente e/ou responsável(eis) pelo atendimento durante o período de internação. grupos:

A população em estudo foi dividida em dois

- gestante adolescente: mulher de idade inferior a 20 anos, segundo definição cronológica do "Comitê de Peritos da OMS",

- gestante adulta: mulher de idade igual ou superior a 20 anos.

A tabulação dos dados foi processada pelo Sistema SPSS ("Statistical Package for the Social Sciences") 8 .

Todos os gráficos apresentados se referem ao período de $01 / 05 / 86$ a 31/07/86 e à população de gestantes do serviço de obstetrícia da área de estudo.

\section{RESULTADOS E DISCUSSĀO}

Comoé descrito na literatura, o objetivo da assistência pré-natal é promover, proteger e recuperar a saúde da gestante e do concepto $3,7,10,11$

Em relação à nossa população de estudo, $22,2 \%$ eram adolescentes e o restante do grupo $(\mathbf{7 7 , 8 \% )}$ eram gestantes com idade maior ou igual a 20 anos que terminaram sua gestação em parto. A cobertura da assistência pré-natal dessas gestantes, no período de estudo, foi de $80,3 \%$ nas gestantes adultas e de $72,9 \%$ nas adolescentes.

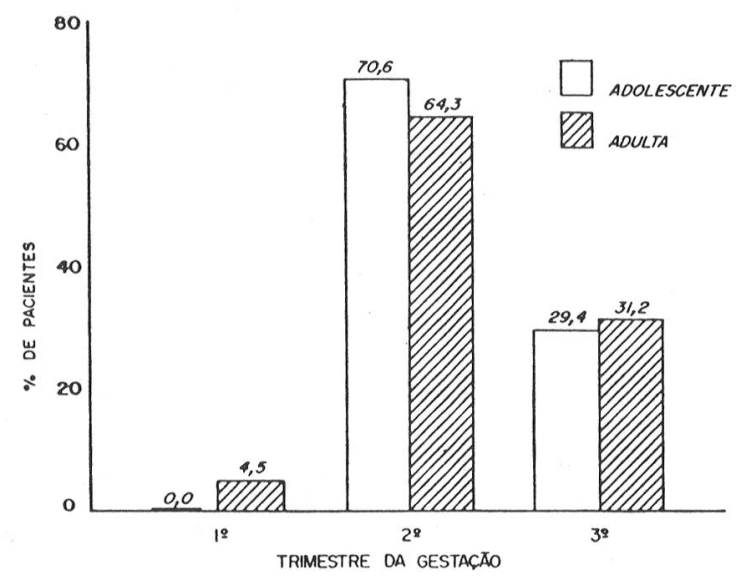

Fig. 1 - Início da assistencia pré-natal segundo grupo etário das gestantes.

A maioria das gestantes, como se observa na Fig. 1, deu entrada no serviço de assistência prénatal no segundo trimestre $(64,3 \%$ das gestantes adultas e 70,6\% das adolescentes) chamando a atenção o fato de nenhuma adolescente ter iniciado o pré-natal precocemente (primeiro trimes. tre), o que não poderia estar indicando: desconhecimento da gravidez; não aceitação da gravidez; pouca importância atribuída a uma assistência pré-natal precoce ou desconhecimento parcial dos serviços de saúde.

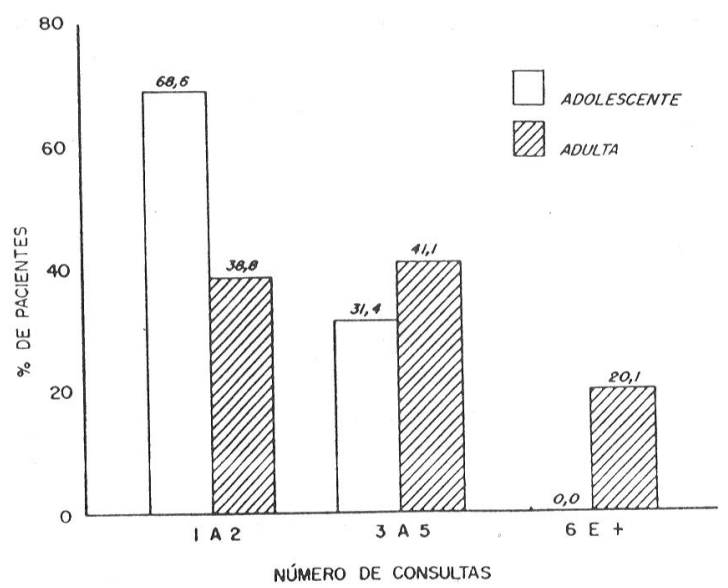

Fig. 2 - Concentração de consultas segundo grupo etário das gestantes. 
Em relação à concentração de consultas (Fig. 2), observamos que a grande maioria das gestantes adolescentes $(68,6 \%)$ teve de uma a duas consultas durante o período pré-natal, sendo que apenas $31,4 \%$ das mesmas tiveram de 3 a 5 consultas, o que responderia ao mínimo preconizado pelo Sub-Programa de Saúde da Mulher da Secretaria de Saúde do Estado de São Paulo ${ }^{11}$.

Observamos, ainda, que a concentração de 6 e mais consultas ocorreu apenas entre as mulheres adultas e em $20,1 \%$ dos casos.

Uma vez que um dos objetivos programáticos da assistência integral à saúde da mulher é aumentar a cobertura e concentração do atendimento pré-natal, proporcionando iguais oportunidades de utilização desses serviços a toda a população ${ }^{7}$, verificamos que nesse serviço, contrariando esta expectativa, a maioria da população de gestantes não recebeu o mínimo esperado para a proteção da mãe e do concepto, fato que se torna ainda mais alarmante no caso das adoles. centes, objeto de nosso estudo.

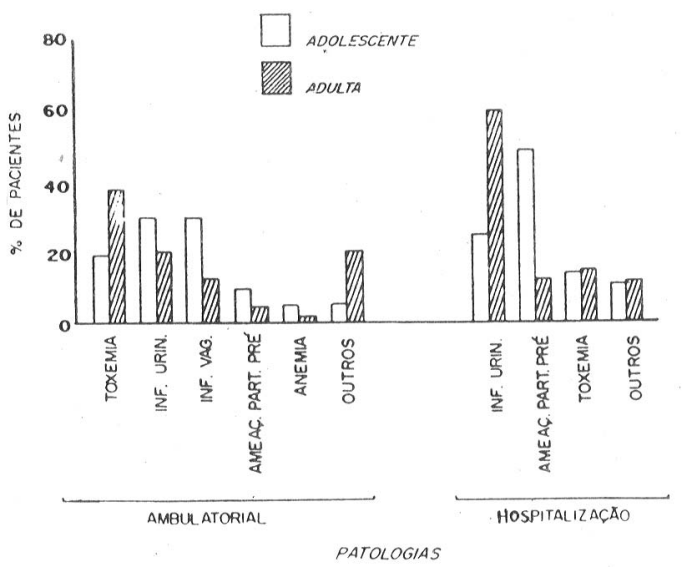

Fig. 3 - Patologias apresentadas no pré-natal com tratamento ambulatorial e hospitalização.

Em relação às patologias apresentadas no pré-natal, encontramos que quase a metade das gestantes adultas $(44,4 \%)$ e mais de um terço das gestantes adolescentes $(39,3 \%)$ tiveram pelo menos um tratamento ambulatorial (Fig. 3).

Pode-se notar, ainda (Fig. 3), que a toxemia foi a patologia mais diagnosticada com tratamento ambulatorial, sendo maior nas gestantes adultas $(38,3 \%)$ que nas adolescentes $(19,9 \%)$, podendo-se aventar como causa provável deste resultado a baixa concentração de consultas que tiveram as adolescentes no pré-natal, não permitindo, como conseqüência, o diagnóstico precoce da mesma.

Em relação às outras patologias, seguem-se a infecção urinária, as infecções vaginais, a ameaça de parto prematuro e as anemias, patologias estas com maior incidência no grupo de gestantes adolescentes, o que confirma achados de outros autores $^{1,2,4}$. A assistência pré-natal serviu, também, para pesquisar patologias (infeçōes vaginais e anemias) que poderiam ser tratadas em nível de controle de saúde da mulher nos períodos pré-concepcional ou intergestacional. Chama a atenção o fato de não se ter registro de outras patologias proprias ou associadas com a gestação, tais como: ameaça de aborto, hemorragias do terceiro trimestre; diabetes mellitus e sífilis, doenças estas de prevalência importante na população.

Ainda em nosso estudo, observamos que $14,7 \%$ das gestantes adultas e $7,9 \%$ das adolescentes foram hospitalizadas durante o pré-natal. As patologias de maior incidencia foram: a infecção urinária nas gestantes adultas $(60,6 \%)$ e ameaça de parto prematuro nas gestantes adolescentes (49,4\%) (Fig. 3).

E importante ressaltar que todas as causas que levaram tanto ao tratamento ambulatorial como ao hospitalar foram patologias evitáveis em nivel de prevenção na assisténcia pré-natal.

Como já foi mencionado, $22,2 \%$ da popula. ção de gestantes estudada eram adolescentes, sendo que, praticamente a metade destas $(48,6 \%)$ terminaram a gestação em parto operatório, $25,7 \%$ por fórceps e $22,9 \%$ por cesárea. Das gestantes adultas, $34,4 \%$ terminaram a gestação em parto operatório, sendo $14,7 \%$ por fórceps e $19,7 \%$ por cesárea.

A proporção de gestantes adolescentes $(22,2 \%)$ reflete uma característica de países em desenvolvimento, segundo a OMS $-1984^{10}$, e os resultados da gestação mostraram niveis maiores de morbimortalidade materna e perinatal, o que indica a necessidade de ações de saúde mais específicas dirigidas a esse grupo considerado de alto risco $^{5,7}$.

Obtivemos, ainda na população de estudo como um todo, que a taxa de cesárea foi de $20,3 \%$, considerada baixar se comparada com a taxa do Estado de São Paulo que é de 43,7\%, mas acima do padrão preconizado pela OMS, que é de $10 \mathrm{a}$ $15 \%^{7}$.

Em relação ds intercorrências no parto, registradas nos prontuários clínicos, durante esse 
período, observamos que estas foram maiores para as adolescentes $(12,9 \%$ para as gestantes adolescentes, $8,2 \%$ para as gestantes adultas). As hemorragias corresponderam a 78,1\%; a toxemia a $\mathbf{1 8 , 8 \%}$ e os acidentes anestesicos a $.3,1 \%$, dentre as intercorrências.

Das gestantes que tiveram hemorragia, 91,5\% eram adultas, o que poderia estar relacionado a uma alta paridade neste grupo etário, tendo em vista esta intercorrência ter como fator predisponente a multiparidade ${ }^{3}$. A alta incidéncia desta condição serve como indicador para avaliar a qualidade da assistência à mulher neste período.

A toxemia, "patologia que está intimamente relacionada com a falta e/ou falha de atenção pré-natal"1, teve maior incidência nas gestantes adolescentes $(44,2 \%)$ que nas gestantes adultas $(8,5 \%)$, levando a crer que só na assistêntia ao parto foi que se detectou as patologias que deveriam ter sido diagnosticadas e tratadas em nível de assistência pré-natal.

A hemorragia e a toxemia contribuíram com $96,9 \%$ das, intercorrencias no parto, sendo que estas patologias são quase sempre controláveis, dependendo da quantidade e qualidade da assistencia no ciclo gravídico-puerperal.

No puerpério, $11,7 \%$ das gestantes apresentaram pelo menos uma patologia, sendo que as gestantes adolescentes tiveram uma maior pro-

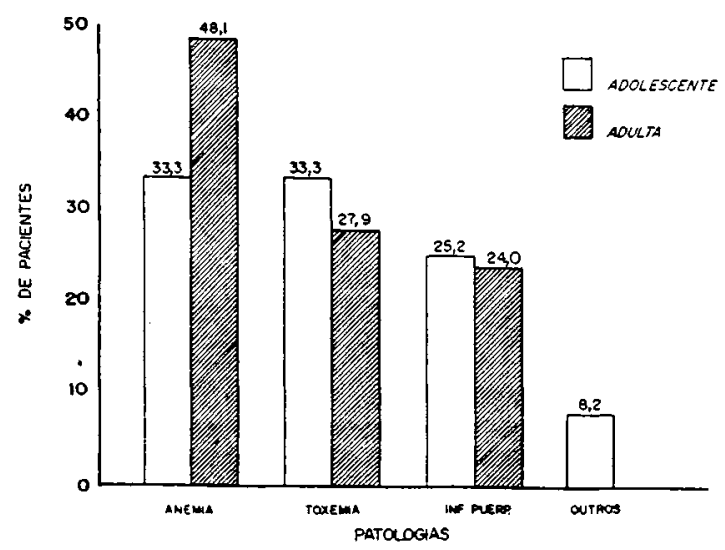

Fig. 4-Intercorrências no puerpério segundo grupo etário das gestantes. porção de intercorrências $(17,1 \%)$ que as gestantes adultas $(10,4 \%)$, sendo a anemia pós-parto, a toxemia e a infecção puerperal patologia diretamente relacionada com a hemorragia intra-parto, as patologias de maior incidência neste período (Fig. 4).

A anemia pós-parto foi maior nas gestantes adultas $(48,1 \%)$. É importante ressaltar que, no serviço, o hemograma era realizado somente em mulheres que apresentassem hemorragia intraparto, o que leva a um sub-diagnóstico da freqüência de anemia.

Assim como no parto, a toxemia continua sendo a segunđa causa de intercorrência obstétrica no puerpério, aparecendo, em seguida, uma patologia própria deste período - a infecção puerperal-, diretamente relacionada com a assistência intra-hospitalar, o que nos leva a questionar a qualidade da assistência oferecida à população em estudo.

Com base nestes dados, aventamos a possibilidade de essas patologias (toxemia, hemorragia e infecção puerperal) estarem refletindo os fatores predisponentes das gestantes bem como a qualidade da assistência oferecida à população em cada período da gestação, uma vez que as mesmas são controláveis ou evitáveis em nível de assistencia global à saúde da mulher e do seu concepto.

\section{CONCLUSÕES}

As gestantes adolescentes do serviço em estudo, apresentaram uma baixíssima concentração de consultas no pré-natal, o que poderia estar sendo relacionado com uma maior proporção de partos operatórios e intercorrências no parto e no puerpério. Portanto, a influência do fator ida. de - que em nossa população em estudo vem acompanhada da falta de assistência a necessidades psicossociais e qualidade de assistência à saúde oferecida pelos serviços -, faz com que as adolescentes apresentem durante a gestação, para si e para o concepto, maior risco reprodutivo.

Sugerimos um maior aprofundamento desta problemática de saúde junto à populaçāo estudada, a fim de se atender, realmente, as necessidades de saúde deste grupo etário. 
LOPEZ A., F.V. et al.; [Adolescent pregnancy: a comparative study]. Rev. Saúde públ, S. Paulo, 23:473-7, 1989.

ABSTRACT: A population of 349 women who gave birth, in an Obstetric Clinic in Greater S. Paulo, Brazil, during the period from $01 / 09 / 86$ to $31 / 07 / 86$ were studied. The results show that the group of pregnant adolescents $(22.2 \%)$ received inadequate prenatal assistance, as the large majority of them (70.6\%) started consultations during the second trimester of their pregnancy, and had an average of two consultations. During pregnancy this group presented a lower incidence of pathological disorders leading to out-patient and hospital treatment ( $39.3 \%$ and $7.9 \%$, respectively) than did the adult pregnant women ( $44.4 \%$ and $14.7 \%$, respectively). With regard to the type of delivery, the adolescents underwent a larger proportion of surgical deliveries, $25.7 \%$ of forceps births and $22.9 \%$ of cesarian sections, as compared with $14.7 \%$ and $19.7 \%$, respectively, for the adult pregnant women. A larger proportion of intra-parturition intercurrencies was registered for the pregnant adolescents ( $12.9 \%$, as against $8.2 \%$ for the adult pregnant women), hemorrhagia and toxemia being the most important. During the puerperium there were complications in $15.7 \%$ of the pregnant adolescents and $11.8 \%$ of the adults post partum anemia, toxemia and puerperal infection being the commonest disorders.

KEYWORDS:Pregnancy in adolescence. Prenatal'care. Pregnancy complications.

\section{REFERÊNCIAS BIBLIOGRÁFICAS}

1. BELEZA FILHO, A.A.L. Gestação na adolescência. J. bras. Ginec., 94:271-4, 1984.

2. CABRAL, A.C.V. et al. Gravidez na adolescencia. J. bras. Ginec., 95:251-3, 1985.

3. EFEITOS do parto sobre a saude materna. Popul. Rep. Ser. J. (8), 1975.

4. A FERTILIDADE na adolescência: riscos e conseqüéncias. Popul Rep. Ser. J., (10), 1976.

5. FRIEDMAN, H.L. \& EDSTROH, K.G. Higiene de la reprodución en la adolescencia: procedimiento para la planificacion de investigaciones sobre senvicios de salud Ginebra, Organizacion Mundial de la Salud, 1983. (OMS - Publicacion Off Set, 77).

6. MENKEN, J.A The health and social consequences of teenage childbaring. Fam Plann. Perspect, 4:45, 1972.

7. MINISTÉRIO DA SAÚDE. Assistência integral d saúde da mulher: bases de ação programatica. Brasinia, Centro de Documentação do Ministério da Saúde, 1984.

8. NIE, N. et al. SPSS: Statistical Package for the Social
Sciences. $2^{\text {nd }}$ ed. New York, McGraw-Hill, 1975.

9. ORGANIZACION MUNDLAL DE LA SALUD. Comite de Expertosi en Necesidades de Salud de los Adolescentes, Ginebra, 1976. Informe. Ginebra, 1977. (Serie de Informes Tecnicos, 609).

10. PROTECCION, promoción de la salud de determinados grupos de población. In: Organizacion Mundial de la Salud. Actividades de la OMS 1982-1983: informe bienal del director general a la Asamblea Mundial de la Saludy a las Naciones Unidas. Ginebra, 1984. p. 99-123.

11. SECRETARIA DE ESTADO DA SAÚDE. Grupo de Saúde da Mulher. Sub-programa de Saúde da Mulher. pré-natal normal. São Paulo, 1986. [Mimeografado.]

12. VITTELO, N. Gestação na adolescéncia: atualização. Femina, (jul): 527-32, 1981.

Recebido para publicaçāo em 2/6/1989 Reapresentado en $2819 / 1989$ Aprovado para publicą̧āo em 6/10/1989 